\title{
Mechanical-Bending-Induced Fluorescence Enhancement in Plastically Flexible Crystals of a GFP Chromophore Analogue
}

\author{
Biswajit Bhattacharya1, Debjit Roy ${ }^{1}$, Somnath Dey ${ }^{1}$, Anisha Puthuvakkal ${ }^{3}$, Surojit Bhunia ${ }^{1,2}$, Saikat Mondal ${ }^{1,2}$, \\ Rituparno Chowdhury ${ }^{1}$, Manjima Bhattacharya ${ }^{1}$, Mrinal Mandal ${ }^{1}$, Kochunnoonny Manoj $^{3}$, Prasun K. Mandal ${ }^{1,2}$ and \\ C. Malla Reddy ${ }^{1,2}$
}

\section{${ }^{1}$ Department of Chemical Sciences, Indian Institute of Science Education and Research (IISER) Kolkata 741246, Nadia, West Bengal (India) \\ ${ }^{2}$ Centre for Advanced Functional Materials (CAFM), Indian Institute of Science Education and Research (IISER) Kolkata 741246, Nadia, West Bengal (India) \\ ${ }^{3}$ Photosciences and Photonics, Chemical Sciences and Technology Division, CSIR-National Institute for Interdisciplinary Science and Technology (CSIR-NIIST), Thiruvananthapuram 695019, India}

prasunchem@iiserkol.ac.in(PKM),cmallareddy@gmail.com(CMR),mondalsaikat334@gmail.com

Single crystals of optoelectronic materials that respond to external stimuli, such as mechanical, light or heat are immensely attractive for next generation smart materials. ${ }^{[1,2]}$ Here we report single crystals of a green fluorescent protein (GFP) chromophore analogue with irreversible mechanical bending and associated unusual enhancement of the fluorescence owing to the suppression of aggregationinduced quenching by aromatic stacked molecules in the perturbed structure. ${ }^{[3]}$ Such fluorescence intensity modulations, which were observed in high-pressure studies earlier, ${ }^{[4]}$ are now shown to occur as function of bending under ambient pressure, hence the study has potential implications for the design of technologically relevant tunable fluorescent materials. ${ }^{[5]}$

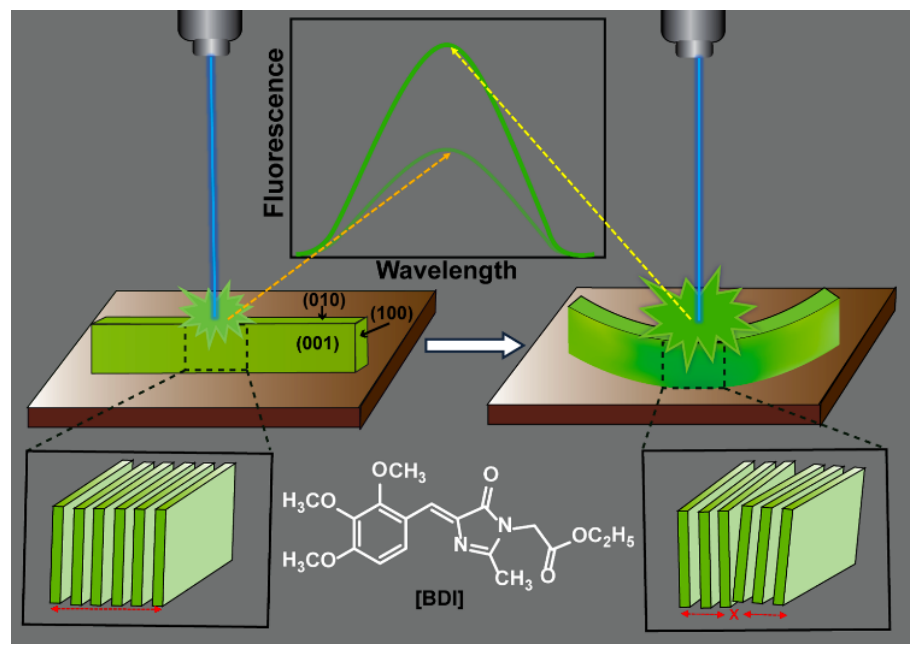

Figure 1. Depiction of fluorescence intensity enhancement in plastically flexible crystals of a GFP chromophore, BDI (bottommiddle) upon mechanical bending due to the perturbation of stacked molecular columns (bottom).

[1] Reddy, C. M., Krishna, G. R. \& Ghosh, S. (2010), CrystEngComm, 12, 2296.

[2] Hayashi, S. \& Koizumi, T. (2016), Angew. Chem. Int. Ed. 55, 2701.

[3] Bhattacharya, B., Roy, D., Dey, S., Puthuvakkal, A., Bhunia, S., Mondal, S., Chowdhury, R., Bhattacharya, M., Mandal, Mrinal., Manoj, K., Mandal, P. K. \& Reddy, C. M. (2020), Angew. Chem. Int. Ed. 59, 19878.

[4] Fabbiani, P. A. \& Pulham, C. R. (2006), Chem. Soc. Rev. 35, 932.

[5] Annadhasan, M., Agrawal, A. R., Bhunia, S., Pradeep, V. V., Zade, S. S., Reddy, C. M. \& Chandrasekar, R. (2020), Angew. Chem. Int. Ed. 59, 13852.

Keywords: Flexible Crystal; Green Fluorescent Protein Chromophore; Mechanical Property; Fluorescence Properties; $\pi-$ Stacking Interactions.

Acta Cryst. (2021), A77, C1223 